\title{
Stem cells and cancer in the liver
}

\author{
Hideki Taniguchi ${ }^{\mathrm{a}, \mathrm{b}}$ and Tetsuhiro Chiba ${ }^{\mathrm{a}}$ \\ ${ }^{a}$ Department of Regenerative Medicine, Graduate School of Medicine, Yokohama City University, Yokohama \\ 236-0004, Japan \\ ${ }^{\mathrm{b}}$ Research Unit for Organ Regeneration, Center for Developmental Biology, Institute of Physical and Chemical \\ Research (RIKEN), Kobe 650-0047, Japan
}

\section{Introduction}

The presence of somatic stem cells, which have high proliferative capacity, multipotency (capacity to differentiate into multiple mature cells with different functions) and self-renewal capacity (capacity to maintain the stem cells as themselves by asymmetric division) has been gradually revealed in various digestive organs such as intestines and liver. Although researchers used to be interested in somatic stem cells from the perspective of their application in regenerative medicine, they have recently been attracting much attention in cancer research because their association with carcinogenesis process was elucidated.

A number of genes that are involved in self-renewal capacity, namely, "stemness genes", have been identified through characterization of hematopoietic stem cells and neural stem cells. These genes were found to be involved in the self-renewal of somatic stem cells also in the liver. On the other hand, because these "stemness genes" turned out to be cancer-associated genes, efforts have been made to understand the carcinogenesis and the mechanisms of cancer recurrence and metastasis from the perspective of hierarchy of stem cell system. The hypothesis that the stem/progenitor cells in tissue are the source of cancer development have started to be verified by prospective analysis for tumor initiation using isolated normal stem/progenitor cells.

In other words, the concept that, as is the case with the presence of stem cells in normal tissues, cancer stem cells are also present in tumor tissues. Cancer stem cells initiate tumors forming a hierarchy of heterogeneous cancer cells.
Here, I will describe the newest findings on hepatic stem/progenitor cells that are attracting attention as a source of the development of hepatocellular carcinoma and cholangiocarcinioma and discuss the association between the self-renewal of hepatic stem cells and the process of malignant transformation.

\section{Hepatic stem cells in the liver}

Hepatic stem cells that are present only in small numbers and are difficult to distinguish by their morphology have been separated and identified from the isolated populations of hepatocytes and cholangiocytes by the highly accurate cell separation method using fluorescence activated cell sorting (FACS, established as a way to purify the hematopoietic stem cells that are present in extremely small numbers in individual organisms) and fluorescent-labeled monoclonal antibodies [1,2].

As an indicator in cell fractionation by FACS, $\alpha$ $6 \beta 1$ integrin has become a focus of attention, which is a receptor specific to laminin, a component of extracellular matrix that is present exclusively in periportal area. This is because hepatic stem cells are hypothesized to be present around the duct of Hering in the portal area of hepatic lobules. The cells with the capacity to form clonal colonies and multipotency to differentiate into hepatocytes and cholangiocytes was shown to be present exclusively in the population of c-Met+c-Kit-CD49f+/lowCD29+CD45TER119-cells. c-Met+c-Kit-CD49f+/lowCD29+CD45 -TER119-cells account for extremely small proportion (only $0.3 \%$ ) of ED (embryonic day) 13.5 fetal liver, in the examination of non-blood cell fraction (CD45- 
Ter119-cells) in fetal liver by using the expression of $\alpha 6$ integrin (CD49f), $\beta 1$ integrin (CD29), c-Kit (stem cell factor receptor) and c-Met (hepatocyte growth factor receptor) as indicators (Fig. 1A-B). Furthermore, experiments by repeating clone sorting clearly verified that some of these cells can proliferate in vitro for more than six months while maintaining their multipotency, suggesting that they have self-renewal capacity which is an important property of stem cells [2]. These cells can remodel hepatic lobular structure and bile duct structure in vivo for prolonged period of time after cell transplantation into the injured liver (Fig. 1C). Moreover, as somatic stem cells are now thought to have higher differentiation plasticity than they were speculated before, c-Met+c-Kit-CD49f+/lowCD29+CD45TER119-cells have demonstrated their potential capacity to differentiate into the pancreas and small intestine that are close to the liver in their embryologic origins [2].

In the future, the mechanism of the regulation of hepatic stem cell differentiation is expected to be elucidated by using the prospective analysis system on these cells, advancing the understanding of the stem cell system in the liver. In addition, it is crucial to scientifically verify the importance of these hepatic stem cells as a source of the development of hepatocellular carcinoma and cholangiocarcinoma.

\section{Self-renewal of stem cells and carcinogenesis}

In regard to hepatocellular carcinoma or intrahepatic cholangiocarcinoma, the carcinogenesis process starting from "oval cells", which have bipotent differentiation capability into hepatocytes and cholangiocytes, has been suggested, and "stem cell hit theory", the hypothesis that stem/progenitor cells in normal tissue are the source of cancer development, has been propounded $[3,4]$. In clinical pathology, combined hepatocellular and cholangiocarcinoma, which has a property between liver and biliary tract cancer, is known to exist. Thus, the carcinogenesis process in the liver that starts from stem/progenitor cells as a source of cancer development is thought to be possible $[5,6]$.

On the other hand, as a new finding in stem cell biology, "stemness genes", which are various gene clusters that are related to the characteristics of stem cells, are being identified. Specifically, molecules in Wnt/ $\beta$ catenin, Hedgehog, Notch and other signaling pathways are being identified as families of molecules that are closely involved in self-renewal of stem cells in hematopoietic stem cells and neural stem cells [7]. Because abnormalities of signaling pathways associated with the similar families of molecules are found with high frequency in leukemia or brain tumor [8,9], it is suggested that these abnormalities would potentially be involved in hyperplasia of tissue or carcinogenesis through excessive self-renewal of somatic stem cells. In fact, it has been reported that activation of $\mathrm{Wnt} / \beta$ catenin signaling induces excessive self-renewal of hematopoietic stem cells, thus leading to the development of leukemia and that activation of polycomb group gene Bmi1, which is involved in self-renewal of neural stem cells, by Sonic hedgehog signaling is important in the development of brain tumor $[10,11]$.

There are a number of reports on mutation of the genes for Wnt/ $\beta$ catenin, Hedgehog and Notch signaling pathway also in liver tumor. Especially in hepatoblastoma, mutation of the $\beta$-catenin gene has been reported to be found in extremely high frequency [12]. In addition, in the pancreas which is embryologically close to the liver, abnormal activation of Hedgehog signaling pathway is shown to be involved in the carcinogenesis in pancreatic cancer [13], suggesting that the families of molecules that are involved in the selfrenewal of stem cells can be closely involved in the carcinogenesis through abnormal self-renewal of somatic stem cells, also in the liver and the pancreas.

\section{Enhanced self-renewal of hepatic stem cells drives cancer initiation}

An excessive and persistent self-renewal signal is one of the key events involved in the initial stages of carcinogenesis [14]. This has been clearly demonstrated in the hematopoietic system, in which oncogenic events including BCR-ABL translocation and constitutive activation of STAT5, enhance the self-renewal of HSCs and establish chronic myeloproliferative disorders $[15,16]$. It is believed that rare transforming events tend to occur in stem cells since they are the only cells that self-renew throughout the lifetime. In fact, increasing evidences support the idea that stem cells and their immediate progeny may be the primary targets of transformation.

In the present study, using highly purified hepatic stem/progenitor cells and clonal analyses, we directly demonstrated that dysregulated self-renewal drives transformation of hepatic stem/progenitor cells (c$\mathrm{Kit}^{-}{ }^{-} \mathrm{CD} 49 \mathrm{f}^{+} \mathrm{CD}^{29}{ }^{+} \mathrm{CD} 45^{-}$Ter-119- cells) [17]. We first confirmed that the two major self-renewal reg- 


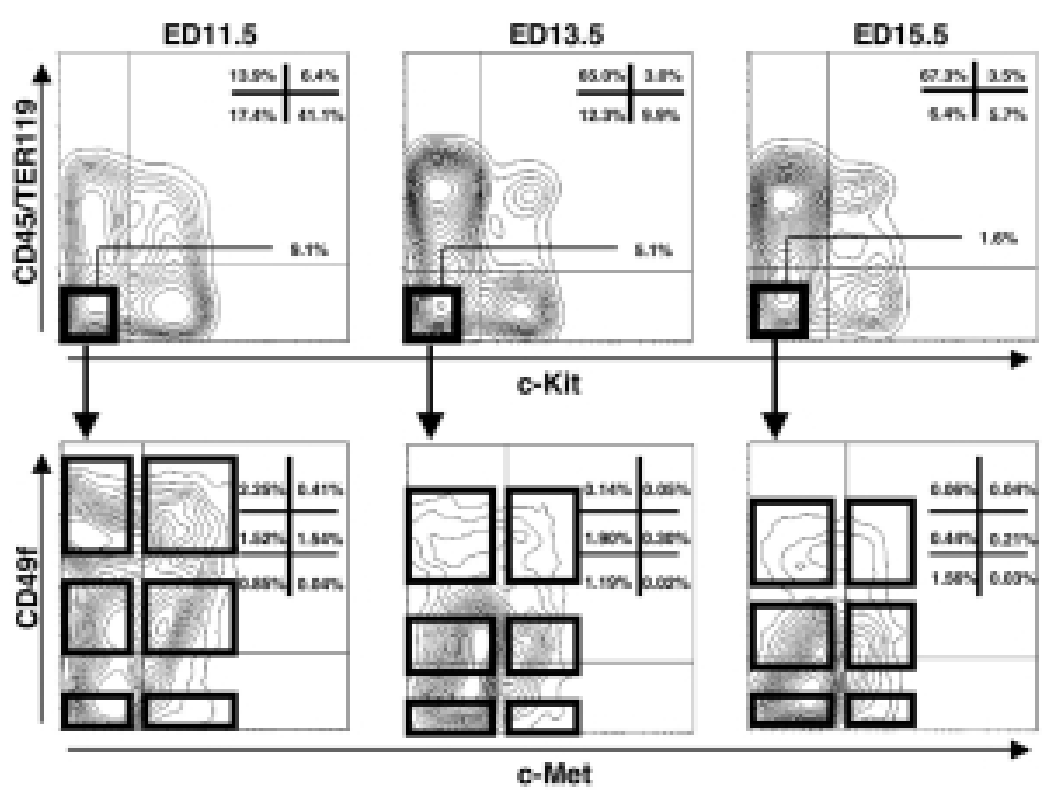

(A)

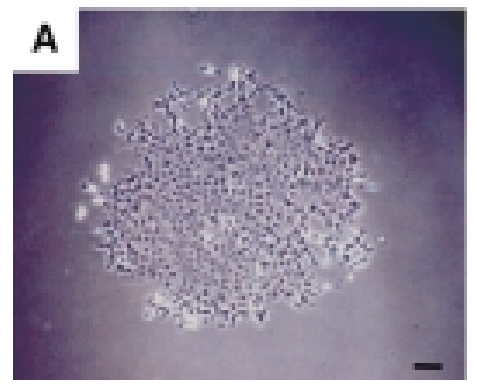

Liver injury

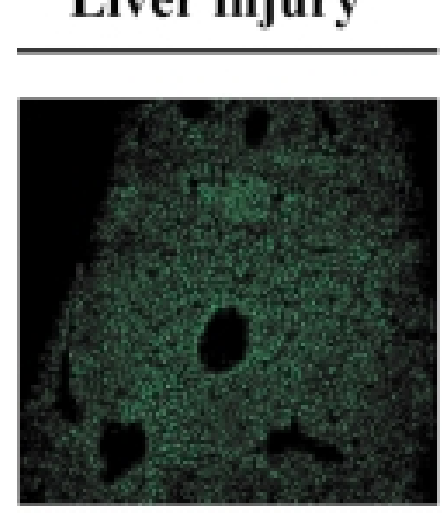

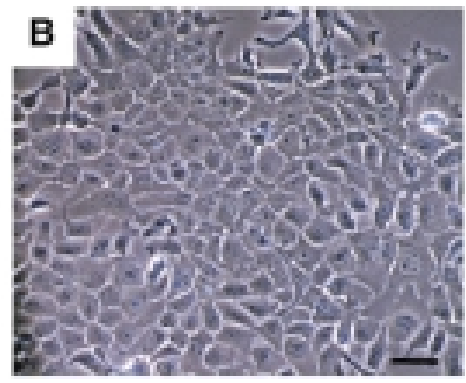

(B)

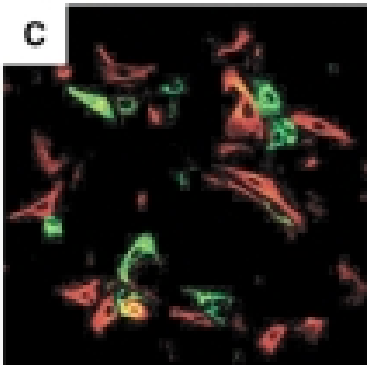

7
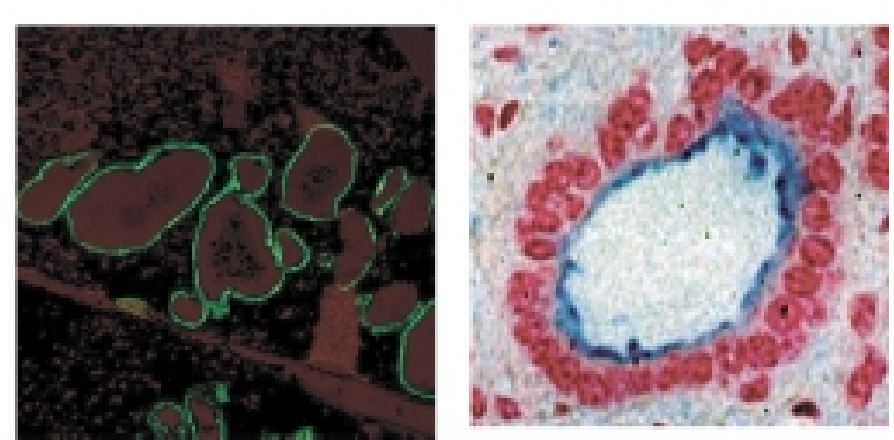

(C)

Fig. 1. (A) Flow cytometric analysis of fetal mouse liver cells (ref2); (B) In vitro multilineage colony formation from a sorted c-Met ${ }^{+} \mathrm{CD} 49 \mathrm{f}^{+/} / \mathrm{low}_{\mathrm{c}-\mathrm{Kit}}{ }^{-} \mathrm{CD} 45^{-} \mathrm{TER}_{119^{-}}$cell (ref2); (C) In vitro liver tissue reconstitution by H-CFU-C. 


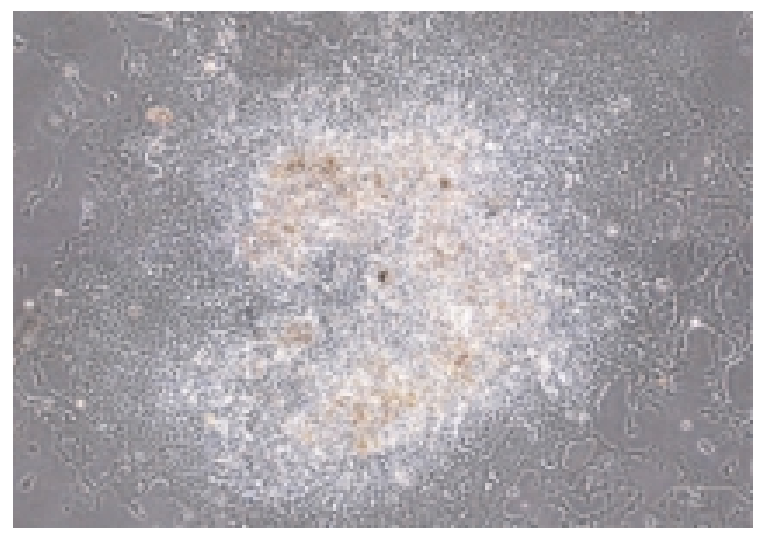

(A)

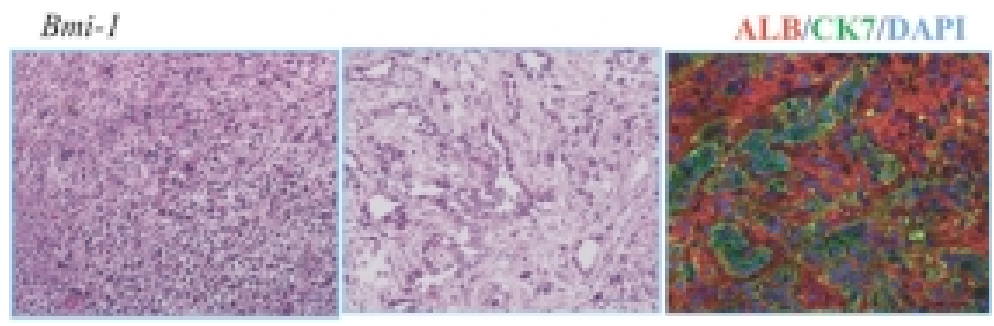

(B)

Fig. 2. (A) Transduction of Bmil into hepatic stem cells gave rise to larger colonies compared with control, which showed a "pile-up" appearance in central area (ref17); (B) Immunohistochemical analysis revealed that the tumors transduced with Bmil consisted of albumint parenchymal cells (red) and $\mathrm{CK}^{+}$glandular structures (green) (Scale bar: $\left.100 \mu \mathrm{m}\right)($ ref17).

ulators of a broad range of stem cells, Bmil and the $\mathrm{Wnt} / \beta$-catenin signaling pathway, also regulate self-renewal of hepatic stem/progenitor cells in culture (Fig. 2A). Intriguingly, their effects were specific to hepatic stem/progenitor cells and did not enhance the colony-forming activity of the other cell fractions. These findings suggest that Bmi1 and active $\beta$-catenin preferentially promote stem cell self-renewal but do not confer a growth advantage or self-renewal capacity on cells with limited growth and differentiation potential. Tumorigenicity of these cells was also assessed by transplantation into non-obese diabetic/severe combined immunodeficient (NOD/scid) mice. Transplantation of Bmi1- or $\beta$-catenin-transduced cells clonally expanded from single hepatic stem/progenitor cells developed tumors (Fig. 2B). The tumors showed both solid proliferation of albumin ${ }^{+}$cells and cytokeratin $7^{+}$duct-like structures corresponding to with welldifferentiated cholangiocarcinoma. The tumor contained focal areas with hepatocyte-like polygonal cells having eosinophilic cytoplasm and importantly several small nests of bipotent cells, expressing both albumin and cytokeratin 7 simultaneously. Spindle-shaped undifferentiated cells with characteristics of sarcoma were also observed in the tumors. The two major components of the tumor, hepatocellular carcinoma (HCC) and cholangiocarcinoma (CC) were intimately intermingled each other and exhibited no clear border, which distinguished the tumor from so called "collision tumor" consisting of HCC and CC. Taken together, these tumors were diagnosed as combined hepatocellular and cholangiocarcinoma (cHCC-CC).

These observations imply that dysregulated selfrenewal of hepatic stem/progenitor cells serves as an early event in hepatocarcinogenesis, and highlight the important roles of Bmil and the $\mathrm{Wnt} / \beta$-catenin signaling pathway in regulating self-renewal of normal and/or cancer stem cells in the liver.

\section{Cancer stem cells in hepatocellular carcinoma}

The new concept of cancer stem cells is based on the idea that stem cells are present also in cancer tissue and a hierarchy of cells is formed, as is the case with normal tissue. Even, in the classical cancer research 
in the 1960's, it was clear that, among cancer cells, only those consisting of certain minor cell populations have tumorigenicity. For example, it was known that, when $10^{6}$ cancer cells are transplanted into nude mice, $10^{6}$ tumors will not be formed, but instead only an extremely small number of tumors will be formed. These cells with tumorigenicity which exist as extremely minor cell populations among all cancer cells are called tumor-initiating cells (T-ICs). In order to explain this phenomenon, two different hypotheses were proposed. One of these is the stochastic model which claims that the cells that comprise a tumor are, in principle, homogeneous and that only those cells in which certain events occurred incidentally turn into T-IC, forming tumors. The other hypothesis is the hierarchy model which claims that the cells that comprise a tumor are, in principle, heterogeneous and that only a part of tumor cells function as T-ICs, forming tumors. This explanation of T-ICs in the hierarchy model provided the basic concept for the idea of cancer stem cells. Cancer stem cells have been identified in leukemia, breast cancer or glioma by separating T-ICs from heterogeneous cancer cell populations.

Also in the human liver cancer cell line, by separating side population (SP) cells using their dye efflux capacity generated by the expression of $\mathrm{ABC}$ transporter as an index, the presence of T-IC for hepatoma exclusively in SP cell fraction has been shown [18]. Specifically, it was shown that the liver cancer stem cells with tumorigenicity and multipotency can be separated and collected by separating SP cells which account for only about $0.25 \%$ (Huh7) or about $0.80 \%$ (PLC/PRF/5) of HCC cell line by using flow cytometry (Fig. 3A-B). Conversely, it was revealed that most HCC cells (nonSP cells) surprisingly do not have tumorigenicity. In vitro studies did not demonstrate much difference in proliferative capacity between SP and non-SP cells, but revealed that SP cells are more resistant to apoptosis than non-SP cells. Moreover, SP cells were found to contain HCC cells that have bipotentiality to differentiate into hepatocytes and cholangiocytes at higher frequency compared to non-SP cells, indicating that SP cells have characteristics that are closer to those of undifferentiated hepatic stem/progenitor cells. The important research theme in the future would be to identify cancer stem cells in clinical samples from liver cancer patients and to elucidate their phenotype and cellular biological properties in detail.

\section{Perspectives}

In terms of hepatocarcinogenesis, it was traditionally believed that long-term, repeated processes between injury and regeneration of damaged mature cells induces accumulation of multiple genetic or epigenetic alterations, which ultimately leads to cancer development $[19,20]$. However, the recent "cancer stem cell hypothesis" implicates the existence of a minor population of cells with self-renewal in the pathogenesis of a variety of cancers, including HCC [21]. Moreover, it is considered that tumor development, at least in some HCC cases, can be attributed to the propagation of the stem/progenitor cell component in hepatocarcinogenesis [22]. Given the close association between inflammation and carcinogenesis, it is reasonable that chronic and persistent tissue injury such as hepatitis viral infection might expand and activate the stem cell pool, thus predisposing the patient to cancer initiation [23].

Currently, the association between the hepatic stem cells present in normal hepatic tissue and cancer stem cells present in liver tumor is not clear, but in many cancers, somatic stem cells are considered to turn into cancer stem cells. Cancer stem cells in many types of liver tumor, however, cannot simply be considered to be derived from hepatic stem/progenitor cells because viral insertion into genome is clearly a direct cause of cancer development in HCC caused by hepatitis B. It should be also assumed that there might be a case in which differentiated hepatocytes regain the trait of stem cells by malignant transformation. Nevertheless, in some types of liver tumor including combined hepatocellular and cholangiocarcinoma, experiments strongly indicated that cancer stem cells were derived from hepatic stem cells. Therefore, in the future, research should be conducted employing prospective experimental system using purified hepatic stem cells, aiming at restructuring the process of malignant transformation to clarify what kind of events should occur in hepatic stem cells for hepatoma or biliary tract cancer to develop. Furthermore, it is crucial to reveal similarity and difference between tissue stem cells in normal tissue and cancer stem cells in cancer tissue by comparing them using the methods including gene expression profiling.

It has been gradually revealed that, in cancer tissue, there is a hierarchical society of cells, which was created based on the systematic cell differentiation and has stem cells on the top, as in the case of normal tissue. Cancer cells that comprise cancer are not homogeneous and therefore, it would be important to realize that these cancer cells constitute a heterogeneous cell population 


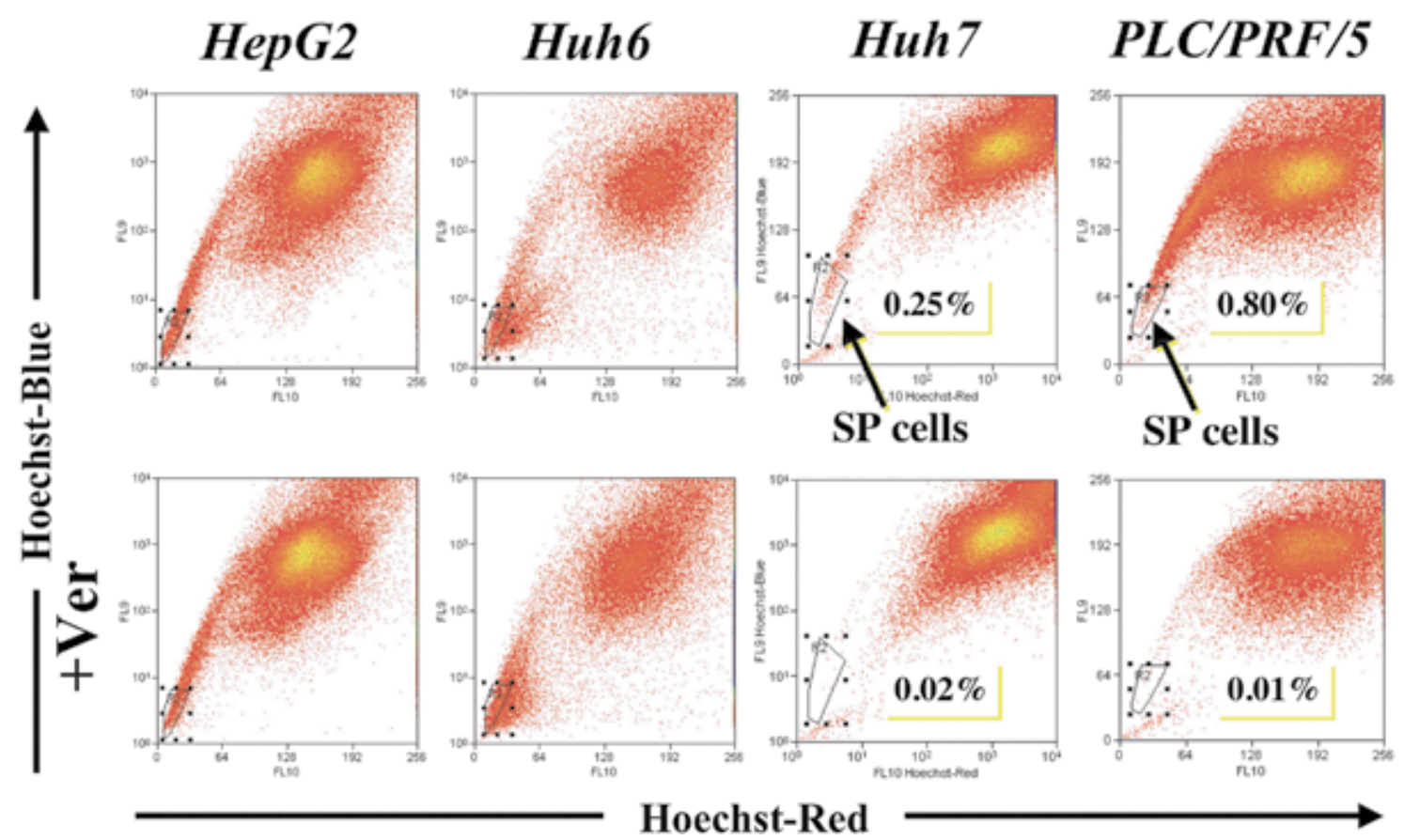

(A)

Huh 7
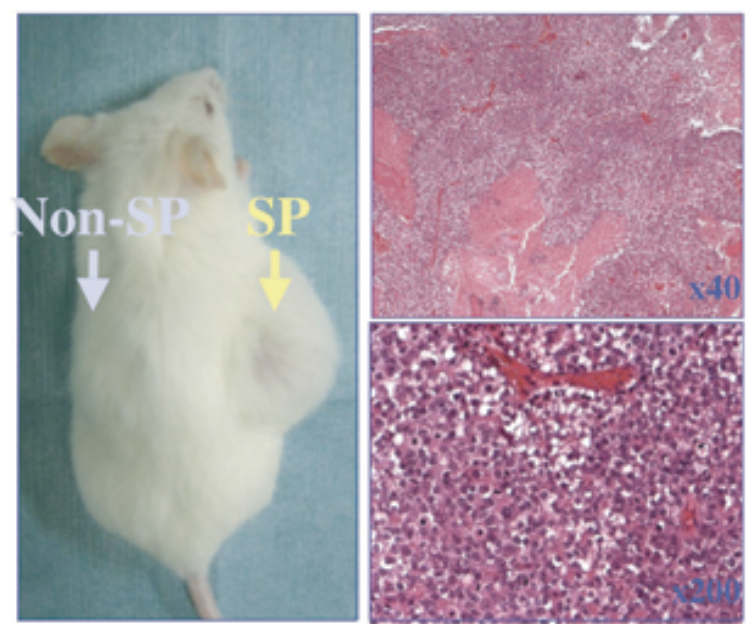

(B)

\section{PLC/PRF/5}

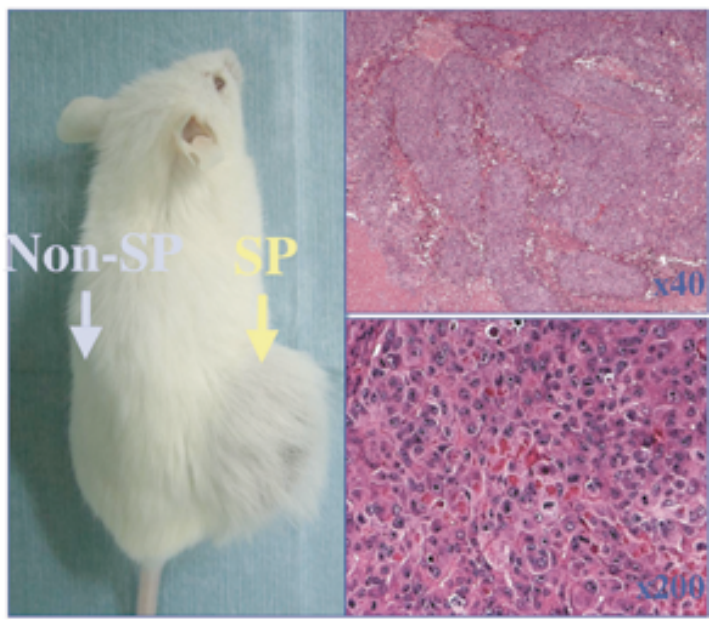

NOD/SCID: 6-10 week-old

Transplanted cells: $1 \times 10^{3}$

16 wks after transplantation

Fig. 3. (A) Existence of SP fraction in liver cancer cell lines (ref18); (B) Assay for the ability of SP/non-SP cells to initiate tumors (ref18). 
with hierarchical property. There is an urgent need to identify cancer stem cells in liver tumor which affects enormous numbers of people and to elucidate their phenotype and cellular biological properties. Furthermore, progress is strongly desired in the research conducted to answer the questions of what kind of events should occur in normal hepatic stem cells or progenitor cells for malignant transformation to happen, and what kind of events should occur in normal hepatocytes for them to regain the property of stem cells (re-programming) as well as other issues, aiming at restructuring the process of malignant transformation by employing prospective experimental system using hepatic stem cells or hepatocytes.

\section{References}

[1] A. Suzuki, Y.W. Zheng, R. Kondo, M. Kusakabe, Y. Takada, K. Fukao, H. Nakauchi and H. Taniguchi, Flowcytometric separation and enrichment of multipotent hepatic progenitor cells in mouse developing liver, Hepatology 32(6) (2000), 1230-1239.

[2] A. Suzuki, Y.W. Zheng, S. Kaneko, M. Onodera, K. Fukao, H. Nakauchi and H. Taniguchi, Clonal identification and characterization of self-renewing pluripotent stem cells in the developing liver, The Journal of Cell Biology 156(1) (2002), 173-184.

[3] H.J. Hacker, P. Steinberg, I. Toshkov et al., Persistence of the cholangiocellular and hepatocellular lesions observed in rats fed a choline-deficient/DL-ethionine-supplemented diet, Carcinogenesis 13 (1992), 271-276.

[4] M.J. Hooth, W.B. Coleman, S.C. Presnell et al., Spontaneous neoplastic transformation of WB-F344 rat liver epithelial cells, Am J Pathol 153 (1998), 1913-1921.

[5] R.A. Allen and J.R. Lisa, Combined liver cell and bile duct carcinoma. Am J Pathol 25 (1949), 647-655.

[6] P.E. Steiner and J. Higginson, Cholangiolocellular carcinoma of the liver, Cancer 12 (1959), 753-759.

[7] T. Reya, S.J. Morrison, M.F. Clarke et al., Stem cells, cancer, and cancer stem cells, Nature 414 (2001), 105-111.

[8] A. Joutel, Tournier-Lasserve E: Notch signalling pathway and human diseases, Semin Cell Dev Biol 9 (1998), 619-625.

[9] R. Wechsler-Reya and M.P. Scott, The developmental biology of brain tumors, Annu Rev Neurosci 24 (2001), 385-428.

[10] T. Reya, A.W. Duncan, L. Ailles et al., A role for Wnt signalling in self-renewal of haematopoietic stem cells, Nature 423 (2003), 409-414.

[11] C. Leung, M. Lingbeek, O. Shakhova et al., Bmil is essential for cerebellar development and is overexpressed in human medulloblastomas, Nature 428 (2004), 337-341.

[12] Y. Wei, M. Fabre, S. Branchereau et al., Activation of beta-catenin in epithelial and mesenchymal hepatoblastomas, Oncogene 19 (2000), 498-504.

[13] D.M. Berman, S.S. Karhadkar, A. Maitra et al., Widespread requirement for Hedgehog ligand stimulation in growth of digestive tract tumours, Nature 425 (2003), 846-851.

[14] M.S. Wicha, S. Liu and G. Dontu, Cancer stem cells: an old idea - a paradigm shift, Cancer Res 66 (2006), 1883-1890.

[15] C.H. Jamieson, I.L. Weissman and E. Passegue, Chronic versus acute myelogenous leukemia: a question of self-renewal, Cancer Cell 6 (2004), 531-533.

[16] Y. Kato, A. Iwama, Y. Tadokoro, K. Shimoda, M. Minoguchi, S. Akira, M. Tanaka, A. Miyajima, T. Kitamura and H. Nakauchi, Selective activation of STAT5 unveils its role in stem cell self-renewal in normal and leukemic hematopoiesis, J Exp Med 202 (2005), 169-179.

[17] T. Chiba, Y.W. Zheng, H. Taniguchi et al., Enhanced selfrenewal capability in hepatic stem/progenitor cells drives cancer initiation, Gastroenterology 133 (2007), 937-950.

[18] T. Chiba, K. Kita, Y.W. Zheng, O. Yokosuka, H. Saisho, A. Iwama, H. Nakauchi and H. Taniguchi, Side Population Purified From Hepatocellular Carcinoma Cells Harbors Cancer Stem Cell-like Properties, Hepatology 44(1) (2005), 240-251.

[19] J. Bruix, L. Boix, M. Sala and J.M. Llovet, Focus on hepatocellular carcinoma, Cancer Cell 5 (2004), 215-219.

[20] L.R. Roberts and G.J. Gores, Hepatocellular carcinoma: molecular pathways and new therapeutic targets, Semin Liver Dis 25 (2005), 212-225.

[21] M. Al-Hajj and M.F. Clarke, Self-renewal and solid tumor stem cells, Oncogene 23 (2004), 7274-7282.

[22] N.D. Theise, J.L. Yao, K. Harada, P. Hytiroglou, B. Portmann, S.N. Thung, W. Tsui, H. Ohta and Y. Nakanuma, Hepatic 'stem cell' malignancies in adults: four cases, Histopathology 43 (2003), 263-271.

[23] P.A. Beachy, S.S. Karhadkar and D.M. Berman, Tissue repair and stem cell renewal in carcinogenesis, Nature 432 (2004), 324-331. 


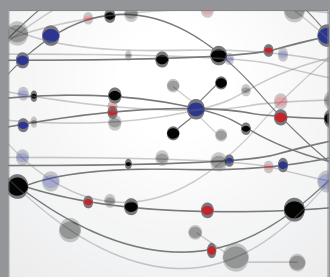

The Scientific World Journal
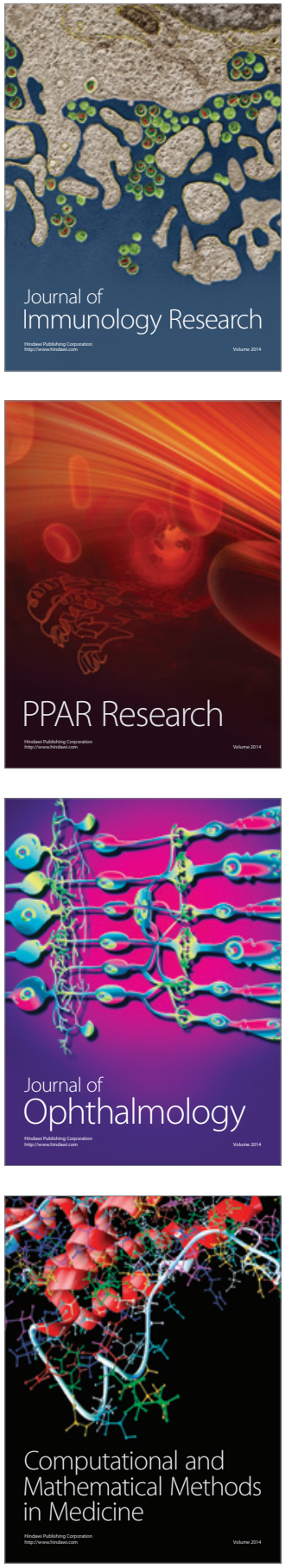

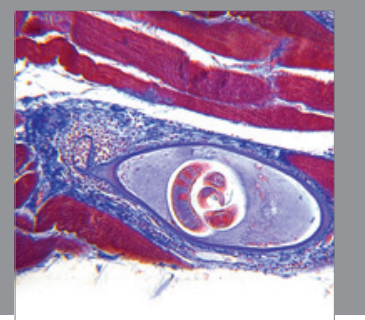

Gastroenterology

Research and Practice
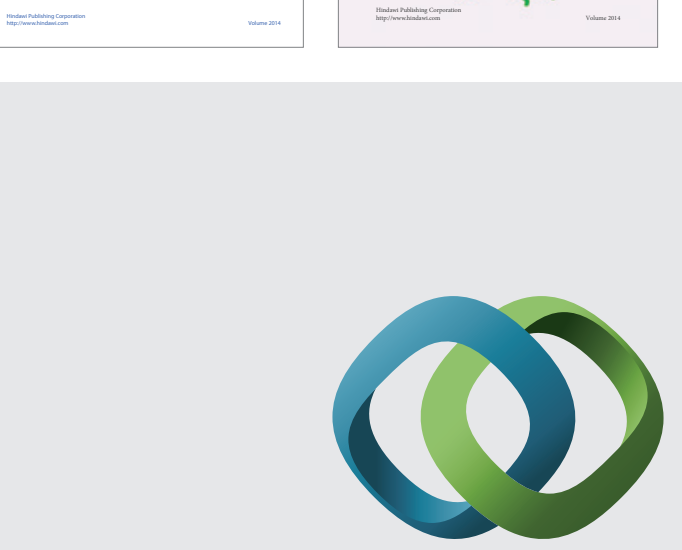

\section{Hindawi}

Submit your manuscripts at

http://www.hindawi.com
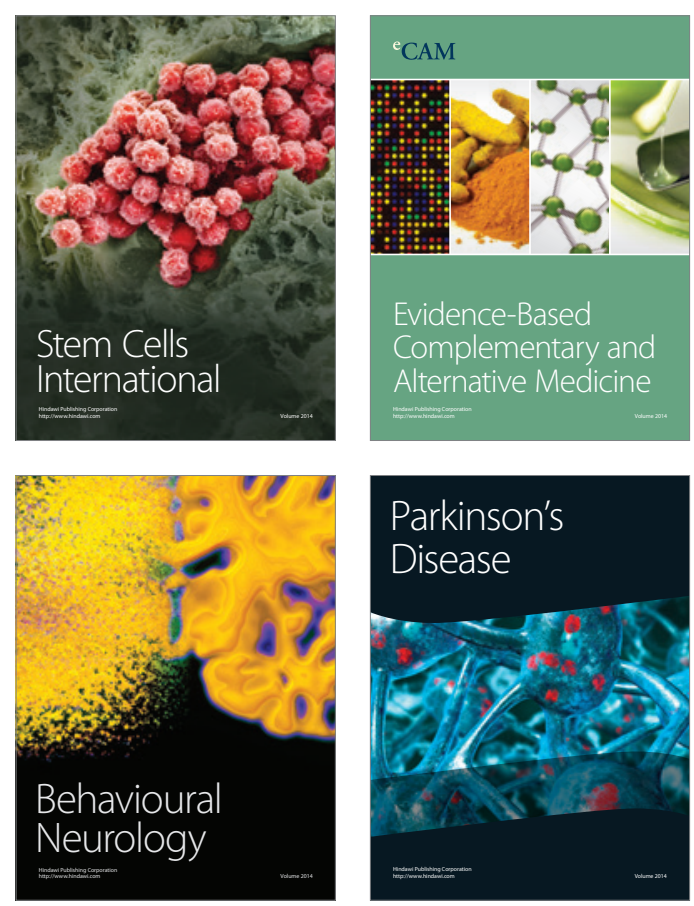

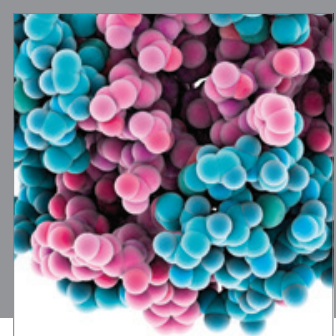

Journal of
Diabetes Research

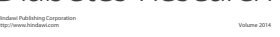

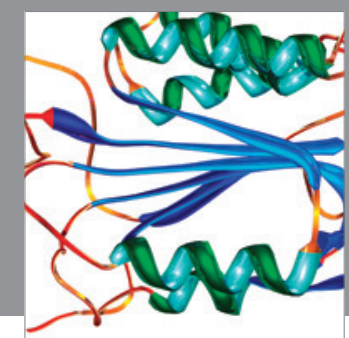

Disease Markers
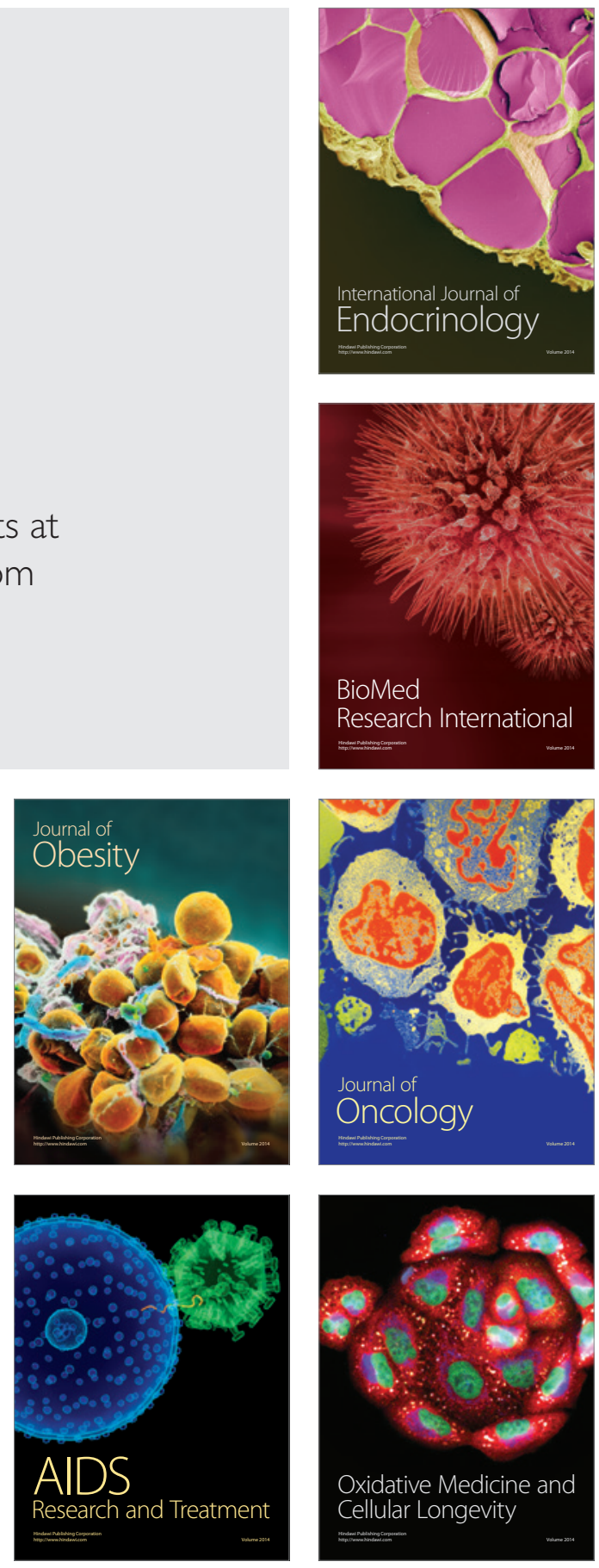